\title{
Nonvanishing modulo $\ell$ of Fourier coefficients of Jacobi forms
}

\author{
Markus Schwagenscheidt
}

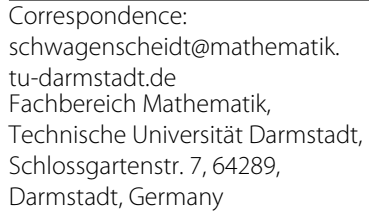

\begin{abstract}
Let $\phi=\sum_{r^{2} \leq 4 m n} c(n, r) q^{n} \zeta^{r}$ be a Jacobi form of weight $k$ (with $k>2$ if $\phi$ is not a cusp form) and index $m$ with integral algebraic coefficients which is an eigenfunction of all Hecke operators $T_{p},(p, m)=1$, and which has at least one nonvanishing coefficient $c\left(n^{*}, r^{*}\right)$ with $r^{*}$ prime to $m$. We prove that for almost all primes $\ell$ there are infinitely many fundamental discriminants $D=r^{2}-4 m n<0$ prime to $m$ with $v_{\ell}(c(n, r))=0$, where $v_{\ell}$ denotes a continuation of the $\ell$-adic valuation on $\mathbb{Q}$ to an algebraic closure. As applications we show indivisibility results for special values of Dirichlet $L$-series and for the central critical values of twisted L-functions of even weight newforms.
\end{abstract}

Keywords: Nonvanishing, Indivisibility, Fourier coefficients, Jacobi forms, Special values of $L$-functions

\section{Introduction and main results}

Let $k$ and $m$ be positive integers. Ono and Skinner [11] and Bruinier [1] have shown, using different methods, that for a non-trivial Hecke eigenform $f=\sum_{n \geq 0} a(n) q^{n}$ of weight $k+1 / 2$ for $\Gamma_{0}(4 m)$ with integral algebraic coefficients, which is not a linear combination of Shimura theta functions, almost all primes $\ell$ have the property that there are infinitely many square free $n$ with $v_{\ell}(a(n))=0$, where $v_{\ell}$ denotes a continuation of the $\ell$-adic valuation on $\mathbb{Q}$ to a fixed algebraic closure.

In this work, we adapt the methods from [1] to prove an analogous result for the Fourier coefficients of Jacobi forms:

Theorem 1.1. Let $\phi \in J_{k, m}$ be a Jacobi form of weight $k$ (with $k>2$ if $\phi$ is not a cusp form) and index $m$ with integral algebraic coefficients $c(n, r)$ which is an eigenform of all Hecke operators $T_{p}$ for $(p, m)=1$. Suppose that there is a discriminant $D^{*}=$ $r^{* 2}-4 m n^{*}<0$ with $\left(D^{*}, m\right)=1$ and $c\left(n^{*}, r^{*}\right) \neq 0$. For almost all primes $\ell$ with $(\ell, 2 m)=1$ there exist infinitely many fundamental discriminants $D=r^{2}-4 m n<0$ prime to $m$ such that $v_{\ell}(c(n, r))=0$.

The finite set of primes $\ell$ for which the theorem does not hold can be described explicitly in terms of the Hecke eigenvalues $\lambda_{p}$ of $\phi$, see (5) and (6) below. The 'bad' primes are essentially those dividing $\lambda_{p}+\left(p^{k-1}+p^{k-2}\right)$ or $\lambda_{p}-\left(p^{k-1}+p^{k-2}\right)$ for all $p$. For cusp forms, the proof that there are only finitely many of these primes relies on

(c) 2016 Schwagenscheidt. Open Access This article is distributed under the terms of the Creative Commons Attribution 4.0 International License (http://creativecommons.org/licenses/by/4.0/), which permits unrestricted use, distribution, and

reproduction in any medium, provided you give appropriate credit to the original author(s) and the source, provide a link to the Creative Commons license, and indicate if changes were made. 
the Shimura correspondence and the elementary estimate $\left|\lambda_{p}\right|<p^{k-1}+p^{k-2}$ for the eigenvalues of integral weight cusp forms from [8].

Further, if $S=\left\{p_{1}, \ldots, p_{s}\right\}$ is a finite set of odd primes which are coprime to $m$ and if $\varepsilon_{1}, \ldots, \varepsilon_{s} \in\{ \pm 1\}$, then we can find (for allmost all primes $\ell$ ) infinitely many fundamental discriminants $D<0$ as in the theorem satisfying the additional local conditions $\left(\frac{D}{p_{j}}\right)=\varepsilon_{j}$ for all $p_{j} \in S$. For a more detailed version of the theorem, which also covers Jacobi forms of higher level, see Theorem 3.5.

The condition $c\left(n^{*}, r^{*}\right) \neq 0$ for some $D^{*}=r^{* 2}-4 m n^{*}<0$ prime to $m$ is obviously necessary, and is violated exactly by the forms in $\sum_{d^{2} \mid m, d>1} J_{k, m / d^{2}} \mid U_{d}$ where $\phi \mid U_{d}(\tau, z)=\phi(\tau, d z)$, compare Lemma 3.1 in [15]. In particular, the theorem is applicable for newforms. Further, for a newform $\phi$ we can replace the requirement of a special discriminant $D^{*}$ as in the theorem by a somewhat nicer looking condition on the Atkin-Lehner eigenvalues of $\phi$, see Proposition 3.6.

The proof of the theorem goes along similar lines as the proof of the corresponding Theorem 4 from [1] for half-integral weight forms. The main ingredients are commutation relations of Hecke operators, Fricke involutions (with regard to the level), and twisting and projection operators for Jacobi forms on the group $\Gamma_{0}\left(N^{2}\right) \ltimes(N \mathbb{Z} \times \mathbb{Z})$, together with the $q$-expansion principle for integral weight modular forms. However, the aforementioned operators for Jacobi forms of higher level seem to be not very common, so it is interesting to see that similar arguments as for half-integral weight modular forms also work for Jacobi forms.

An important tool in the proof of Theorem 1.1 is the following result on the algebraicity of Fourier expansions of a Jacobi form at the cusps, which might be of independent interest.

Theorem 1.2. Let $\phi \in J_{k, m}\left(\Gamma_{1}\left(N^{2}\right) \ltimes(N \mathbb{Z} \times \mathbb{Z})\right)$ have integral algebraic Fourier coefficients $c(n, r)$ and let $\ell$ be a prime with $(\ell, 2 m N)=1$. For $M \in S L_{2}(\mathbb{Z})$ the function $\left.\phi\right|_{k, m} M$ has algebraic Fourier coefficients and it holds $v_{\ell}\left(\left.\phi\right|_{k, m} M\right) \geq v_{\ell}(\phi)$.

Here we wrote $\nu_{\ell}(\phi)=\inf _{n, r} \nu_{\ell}(c(n, r))$ for a Jacobi form $\phi=\sum_{r^{2} \leq 4 m n} c(n, r) q^{n} \zeta^{r}$. For the proof, we apply the $q$-expansion principle to the components of the vector valued modular form corresponding to $\phi$ via its theta decomposition.

Let us now give two applications of Theorem 1.1, both of which are strengthenings of applications from [1]: First, we consider the central critical values of twisted $L$ functions of even weight newforms. By the results of [15], the space of cuspidal Jacobi newforms $J_{k+1, m}^{\text {cusp, new }}$ of weight $k+1$ and index $m$ is isomorphic as a module over the Hecke algebra to the space $S_{2 k}^{\text {new, }-}(m)$ of elliptic newforms of weight $2 k$ and level $m$ whose $L$-function $L(f, s)$ has a minus sign in its functional equation. Let $f \in$ $S_{2 k}^{\text {new, }-}(m)$ be a normalised newform and let $\phi \in J_{k+1, m}^{\text {cusp,new }}$ be the corresponding Jacobi newform with integral algebraic coefficients. If $D=r^{2}-4 m n<0$ with $(D, m)=1$ is a fundamental discriminant which is a square $\bmod 4 m$, the formula of Waldspurger [16] in the explicit form given by Gross, Kohnen and Zagier [6] states that

$$
\frac{|c(n, r)|^{2}}{\langle\phi, \phi\rangle}=\frac{(k-1) !}{2^{2 k-1} \pi^{k} m^{k-1}}|D|^{k-1 / 2} \frac{L(f, D, k)}{\langle f, f\rangle},
$$


where $L(f, D, s)$ denotes the $L$-function of $f$ twisted by the Kronecker symbol $\left(\frac{D}{.}\right)$. Now Theorem 1.1 implies the following result.

Corollary 1.3. For a normalised newform $f \in S_{2 k}^{\text {new,- }}(m)$ there exists a nonzero complex number $\Omega_{f}$ such that for almost all primes $\ell$ with $(\ell, 2 m)=1$ there are infinitely many fundamental discriminants $D<0$ prime to $m$ which are squares mod $4 m$ such that

$$
v_{\ell}\left(\frac{L(f, D, k)|D|^{k-1 / 2}}{\Omega_{f}}\right)=0 .
$$

Note that for a newform $f \in S_{2 k}^{\text {new, }}(m)$ the functional equation of the twisted $L$-function implies $L(f, D, k)=0$ for fundamental discriminants $D<0$ which are coprime to $m$ and squares mod $4 m$. Compared to the very similar Theorem 5 in [1] or Corollary 2 in [11], the fundamental discriminants $D$ in our corollary are now all coprime to $m$ and squares $\bmod 4 m$.

As explained in Corollaries 3 and 4 in [11], a result like Corollary 1.3 has implications for the orders of the Tate-Shafarevich groups of quadratic twists of elliptic curves over $\mathbb{Q}$. For related results, see also [9].

As a second application we consider the coefficients of the Eisenstein series $E_{k, 1} \in$ $J_{k, 1}$ for even $k \geq 4$, which are given by special values of Dirichlet $L$-series. $E_{k, 1}$ is an eigenform of all Hecke operators $T_{p}$ with eigenvalue $\sigma_{2 k-3}(p)=1+p^{2 k-3}$. By Theorem 2.1 in [5], the Fourier coefficients $e_{k, 1}(n, r)$ of $E_{k, 1}$ for fundamental discriminants $D=$ $r^{2}-4 n<0$ are given by

$$
e_{k, 1}(n, r)=\frac{L\left(2-k, \chi_{D}\right)}{\zeta(3-2 k)}
$$

where $\chi_{D}=\left(\frac{D}{.}\right)$. It is well known that $L\left(2-k, \chi_{D}\right)=-B_{k-1, \chi_{D}} /(k-1)$ with the generalised Bernoulli numbers $B_{k-1, \chi_{D}}$, so the values $L\left(2-k, \chi_{D}\right)$ are non-zero rational numbers whose denominators are divisible only by primes $\ell$ such that $(\ell-1)$ divides $2(k-1)$, see [3]. Hence the coefficients of $\zeta(3-2 k) E_{k, 1}$ are $\ell$-integral rational numbers with bounded denominators for almost all primes $\ell$. The same is true for the coefficients of $\zeta(3-2 k) E_{k, 1} \mid V_{m}$, where $V_{m}: J_{k, 1} \rightarrow J_{k, m}$ is the usual Hecke-type operator defined in [5], §4. Moreover, for any $\phi \in J_{k, 1}$ and a fundamental discriminant $D=r^{2}-4 m n<0$ we have $c_{\phi \mid V_{m}}(n, r)=c_{\phi}(m n, r)$ by the explicit formula for the action of $V_{m}$ on the Fourier expansion of $\phi$. Thus Theorem 1.1 applied to a suitable multiple of $\zeta(3-2 k) E_{k, 1} \mid V_{m}$ gives the following result.

Corollary 1.4. Let $m$ be a positive integer and let $k \geq 4$ be an even integer. For almost all primes $\ell$ with $(\ell, 2 m)=1$ there are infinitely many fundamental discriminants $D<0$ prime to $m$ which are squares mod $4 m$ such that

$$
v_{\ell}\left(L\left(2-k, \chi_{D}\right)\right)=0 .
$$

On the other hand, for odd $k$ and negative $D$ the functional equation of $L\left(s, \chi_{D}\right)$ gives $L\left(2-k, \chi_{D}\right)=0$.

Eventually, we remark that the techniques of this paper can be used to prove analogous results for skew-holomorphic Jacobi forms as defined in [14]: Recall that a skew-holomorphic Jacobi form of weight $k$ and index $m$ is a smooth function 
$\phi: \mathbb{H} \times \mathbb{C} \rightarrow \mathbb{C}$ which is holomorphic in z, which is invariant under a modified slash action $\left.\right|_{k, m} ^{*}$ of $S L_{2}(\mathbb{Z}) \ltimes \mathbb{Z}^{2}$ where $(c \tau+d)^{k}$ is replaced by $(c \bar{\tau}+d)^{k-1}|c \tau+d|$ (see below for the slash operator $\left.\right|_{k, m}$ on holomorphic Jacobi forms), and which has a Fourier expansions of the form

$$
\phi(\tau, z)=\sum_{\substack{n, r \in \mathbb{Z} \\ r^{2} \geq 4 m n}} c(n, r) e\left(\frac{r^{2}-4 m n}{2 m} i v\right) q^{n} \zeta^{r}, \quad(\tau=u+i v) .
$$

If we replace $r^{2} \leq 4 m n$ by $r^{2} \geq 4 m n$ and $\left.\right|_{k, m}$ by $\left.\right|_{k, m} ^{*}$, and add a factor $e\left(\frac{r^{2}-4 m n}{2 m} i v\right)$ in the Fourier expansions in all proofs in Section 2, we see that the results in Section 2 are also valid for skew-holomorphic forms.

Since a skew-holomorphic Jacobi form also has a theta decomposition and thereby corresponds to a vector valued modular form (see [2], Section 1.1), the proof of Theorem 1.2 also works for skew-holomorphic forms.

Further, Theorem 3.5 and the propositions and lemmas used for its derivation have counterparts for skew-holomorphic Jacobi forms (just replace $D \leq 0$ with $D \geq 0$ everywhere) and can be proved in the same way. But we have to be careful with the remarks after Theorem 3.5: It seems (to the best knowledge of the author) that the analog of the isomorphism between $J_{k+1, m}^{\text {cusp }}$ with a subspace of $S_{2 k}$ for skewholomorphic Jacobi forms has not been proved in the literature, so estimates for the eigenvalues of skew-holomorphic Jacobi forms are not readily available.

The situation is similar with the Waldspurger formula (1). Although it should be true for skew-holomorphic forms by similar arguments as in the holomorphic case, we could not find a proof in the literature. Assuming the formula, we get an analog of Corollary 1.3 for newforms $f \in S_{2 k}^{\text {new, }}(m)$, now yielding infinitely many positive fundamental discriminants $D=r^{2}-4 m n>0$ with $\nu_{\ell}\left(L(f, D, k)|D|^{k-1 / 2} / \Omega_{f}\right)=0$.

\section{Operators on Jacobi forms of higher level}

For the basic facts about Jacobi forms (of level 1) we refer the reader to the standard reference [5]. In this section we define Hecke operators and the Fricke involution on Jacobi forms for $\Gamma_{0}\left(N^{2}\right) \ltimes(N \mathbb{Z} \times \mathbb{Z})$, as well as certain twisting and projection operators, and we study their commutation relations.

Let $e(x):=e^{2 \pi i x}$ and $e^{m}(x):=e^{2 \pi i m x}$ for $x \in \mathbb{C}$ and $m \in \mathbb{Z}$. The real Jacobi group $S L_{2}(\mathbb{R}) \ltimes \mathbb{R}^{2} \cdot S^{1}$ with group law

$$
[M, X, \xi] \cdot\left[M^{\prime}, X^{\prime}, \xi^{\prime}\right]=\left[M M^{\prime}, X M^{\prime}+X^{\prime}, \xi \xi^{\prime} e\left(\operatorname{det}\left(\begin{array}{c}
X M^{\prime} \\
X^{\prime}
\end{array}\right)\right)\right]
$$

acts on holomorphic functions $\phi: \mathbb{H} \times \mathbb{C} \rightarrow \mathbb{C}$ by

$$
\begin{aligned}
\left.\phi\right|_{k, m}\left[\left(\begin{array}{ll}
a & b \\
c & d
\end{array}\right),[\lambda, \mu], \xi\right](\tau, z) \\
=\xi^{m}(c \tau+d)^{-k} e^{m}\left(-\frac{c(z+\lambda \tau+\mu)^{2}}{c \tau+d}+\lambda^{2} \tau+2 \lambda z+\lambda \mu\right) \\
\quad \times \phi\left(\frac{a \tau+b}{c \tau+d}, \frac{z+\lambda \tau+\mu}{c \tau+d}\right) .
\end{aligned}
$$

To simplify the notation we sometimes write $\left.\phi\right|_{k, m} M$ for $\left.\phi\right|_{k, m}[M, 0,1]$ and $\left.\phi\right|_{m} X$ for $\left.\phi\right|_{k, m}[1, X, 1]$. 
Definition 2.1. Let $\Gamma \subseteq \mathrm{SL}_{2}(\mathbb{Z})$ be a subgroup of finite index, let $\chi$ be a character of $\Gamma$ and let $L \subseteq \mathbb{R}^{2}$ be a rank 2 lattice which is invariant under right multiplication by $\Gamma$. A Jacobi form of weight $k$ and index $m$ for $\Gamma \ltimes L$ with character $\chi$ is a holomorphic function $\phi: \mathbb{H} \times \mathbb{C} \rightarrow \mathbb{C}$ satisfying the following properties:

$\left.1 \phi\right|_{k, m} M=\chi(M) \phi$ for every $M \in \Gamma$.

$\left.2 \phi\right|_{m} X=\phi$ for every $X \in L$.

3 For every $M \in S L_{2}(\mathbb{Z})$, the function $\left.\phi\right|_{k, m} M$ has a Fourier expansion of the form

$$
\sum_{\substack{n, r \in \mathbb{Q} \\ r^{2} \leq 4 m n}} c_{M}(n, r) q^{n} \zeta^{r}, \quad\left(q^{n}=e(n \tau), \zeta^{r}=e(r z)\right),
$$

with coefficients $c_{M}(n, r) \in \mathbb{C}$.

If for all $M \in \mathrm{SL}_{2}(\mathbb{Z})$ we have $c_{M}(n, r)=0$ whenever $r^{2}-4 m n=0$, we call $\phi$ a Jacobi cusp form. The corresponding spaces of Jacobi forms will be denoted by $J_{k, m}(\Gamma \ltimes L, \chi)$ and $J_{k, m}^{\text {cusp }}(\Gamma \ltimes L, \chi)$, and for $\Gamma=\mathrm{SL}_{2}(\mathbb{Z})$ and $L=\mathbb{Z}^{2}$ we just write $J_{k, m}$ and $J_{k, m}^{\text {cusp }}$.

In the following, we will mainly work with Jacobi forms for the group $\Gamma_{0}\left(N^{2}\right) \ltimes$ $(N \mathbb{Z} \times \mathbb{Z})$ and character $\chi(M)=\chi(d)$ for $M=\left(\begin{array}{ll}a & b \\ c & d\end{array}\right) \in \Gamma_{0}\left(N^{2}\right)$, where $N$ is a positive integer and $\chi$ is a Dirichlet character $\bmod N^{2}$. Note that the transformation behaviour of $\phi \in J_{k, m}\left(\Gamma_{0}\left(N^{2}\right) \ltimes(N \mathbb{Z} \times \mathbb{Z}), \chi\right)$ under $N \mathbb{Z} \times \mathbb{Z}$ implies $c(n, r)=c(n+$ $\left.r N \lambda+m N^{2} \lambda^{2}, r+2 m N \lambda\right)$ for all $\lambda \in \mathbb{Z}$.

Let $p$ be a prime. Similarly as in [5], we define the $p$-th Hecke operator on $J_{k, m}\left(\Gamma_{0}\left(N^{2}\right) \ltimes(N \mathbb{Z} \times \mathbb{Z}), \chi\right)$ by

$$
\phi\left|T_{p}=p^{k-4} \sum_{\begin{array}{r}
M \in \Gamma_{0}\left(N^{2}\right) \backslash M_{0}\left(N^{2}\right) \\
\operatorname{det}(M)=p^{2} \\
\operatorname{gcd}(M)=\square
\end{array}} \chi(a) \sum_{\lambda, \mu(p)} \phi\right|_{k, m}\left[\frac{1}{p} M,[N \lambda, \mu], 1\right],
$$

where $M_{0}\left(N^{2}\right)$ is the set of integral $2 \times 2$ matrices whose lower left entry is divisible by $N^{2}$, and $\operatorname{gcd}(M)=\square$ means that the greatest common divisor of the entries of $M$ is a square.

Lemma 2.2. The Hecke operator $T_{p}$ defines an endomorphism of $J_{k, m}\left(\Gamma_{0}\left(N^{2}\right) \ltimes\right.$ $(N \mathbb{Z} \times \mathbb{Z}), \chi)$ which maps cusp forms to cusp forms. The $(n, r)$-th coefficient of $\phi \mid T_{p}=$ $\sum_{r^{2} \leq 4 m n} c^{*}(n, r) q^{n} \zeta^{r}$ is given by

$$
\begin{aligned}
c^{*}(n, r)= & c\left(p^{2} n, p r\right)+\chi(p) C_{p}(n, r, m) p^{k-3} c(n, r) \\
& +\chi\left(p^{2}\right) p^{2 k-3} \sum_{\lambda(p)} c\left(\left(n+r N \lambda+m N^{2} \lambda^{2}\right) / p^{2},(r+2 m N \lambda) / p\right),
\end{aligned}
$$

where

$$
C_{p}(n, r, m)= \begin{cases}p\left(\frac{D}{p}\right), & \text { if } p \nmid m, \\ 0, & \text { if } p \mid m ; \quad p \nmid r, \\ -p, & \text { if } p \mid m, r ; \quad p \nmid n, \\ p(p-1), & \text { if } p \mid m, r, n .\end{cases}
$$

Here we use the convention $c(n, r)=0$ if $n$ or $r$ is not integral. 
Proof. The well-definedness and the mapping property of $T_{p}$ are easy exercises, so we only compute the Fourier expansion of $\phi \mid T_{p}$. As representatives for $\Gamma_{0}\left(N^{2}\right) \backslash$ $M_{0}\left(N^{2}\right)$ with determinant $p^{2}$ and square content we use the matrices

$$
\alpha_{b}=\left(\begin{array}{ll}
1 & b \\
0 & p^{2}
\end{array}\right), 0 \leq b<p^{2}, \quad \beta_{h}=\left(\begin{array}{cc}
p & h \\
0 & p
\end{array}\right), 0<h<p, \quad \sigma=\left(\begin{array}{cc}
p^{2} & 0 \\
0 & 1
\end{array}\right),
$$

compare Theorem 1.7 in [13]. We compute the contribution to $c^{*}(n, r)$ for each of the three types of representatives individually.

We first decompose $\left[\frac{1}{p} \alpha_{b},[N \lambda, \mu], 1\right]$ as

$$
\begin{aligned}
{\left[\left(\begin{array}{cc}
1 / p & b / p \\
0 & p
\end{array}\right),[N \lambda, \mu], 1\right]=} & {\left[\left(\begin{array}{ll}
1 & 0 \\
0 & 1
\end{array}\right),[N \lambda p, 0], 1\right] } \\
& \cdot\left[\left(\begin{array}{cc}
1 / p & b / p \\
0 & p
\end{array}\right),[0, \mu-N \lambda b], 1\right]
\end{aligned}
$$

Since $\phi$ is invariant under the slash operation of the first element on the right-hand side, the $\alpha_{b}$-part of $\phi \mid T_{p}$ is given by

$$
\begin{aligned}
& \left.p^{k-4} \sum_{b\left(p^{2}\right)} \sum_{\lambda, \mu(p)} \phi\right|_{k, m}\left[\left(\begin{array}{cc}
1 / p & b / p \\
0 & p
\end{array}\right),[N \lambda p, 0], 1\right] \\
& =\left.p^{k-4} \sum_{b\left(p^{2}\right)} \sum_{\lambda, \mu(p)} \phi\right|_{k, m}\left[\left(\begin{array}{cc}
1 / p & b / p \\
0 & p
\end{array}\right),[0, \mu-N \lambda b], 1\right] \\
& =p^{k-4} \sum_{b\left(p^{2}\right)} \sum_{\lambda, \mu(p)} p^{-k} \phi\left((\tau+b) / p^{2},(z+\mu-N \lambda b) / p\right) .
\end{aligned}
$$

If we replace $\mu-N \lambda b$ by $\mu$ and plug in the Fourier expansion of $\phi$, the last line becomes

$$
p^{-3} \sum_{b\left(p^{2}\right)} \sum_{\mu(p)} \sum_{\substack{n, r \in \mathbb{Z} \\ r^{2} \leq 4 m n}} c(n, r) e\left(n b / p^{2}\right) e(r \mu / p) q^{n / p^{2}} \zeta^{r / p} .
$$

Now the product of the two exponential sums over $b$ and $\mu$ gives a factor $p^{3}$ if $n$ is divisible by $p^{2}$ and $r$ is divisible by $p$, and vanishes otherwise. Thus we get the contribution

$$
\sum_{\substack{n, r \in \mathbb{Z} \\ r^{2} \leq 4 m n \\ p^{2}|n, p| r}} c(n, r) q^{n / p^{2}} \zeta^{r / p}=\sum_{\substack{n^{\prime}, r^{\prime} \in \mathbb{Z} \\ r^{\prime 2} \leq 4 m n^{\prime}}} c\left(p^{2} n^{\prime}, p r^{\prime}\right) q^{n^{\prime}} \zeta^{r^{\prime}}
$$

of the $\alpha_{b}$-part, where we replaced $n=p^{2} n^{\prime}$ and $r=p r^{\prime}$.

For $\left[\frac{1}{p} \beta_{h},[N \lambda, \mu], 1\right]$ we have

$$
\begin{aligned}
{\left[\left(\begin{array}{cc}
1 & h / p \\
0 & 1
\end{array}\right),[N \lambda, \mu], 1\right]=} & {\left[\left(\begin{array}{ll}
1 & 0 \\
0 & 1
\end{array}\right),[N \lambda, \mu], 1\right] } \\
& \cdot\left[\left(\begin{array}{cc}
1 & h / p \\
0 & 1
\end{array}\right),[0,-N \lambda h / p], e\left(N^{2} \lambda^{2} h / p\right)\right] .
\end{aligned}
$$


Using the invariance of $\phi$ under the slash operation of the first element on the right-hand side we see that the $\beta_{h}$-part of $\phi \mid T_{p}$ equals $\chi(p)$ times

$$
\begin{aligned}
& \left.p^{k-4} \sum_{h(p)^{*} \lambda, \mu(p)} \phi\right|_{k, m}\left[\left(\begin{array}{cc}
1 & h / p \\
0 & 1
\end{array}\right),[N \lambda, \mu], 1\right] \\
& =p^{k-3} \sum_{h(p)^{*} \lambda(p)} \sum_{k, m}\left[\left(\begin{array}{cc}
1 & h / p \\
0 & 1
\end{array}\right),[0,-N \lambda h / p], e\left(N^{2} \lambda^{2} h / p\right)\right] \\
& =p^{k-3} \sum_{h(p)^{*} \lambda(p)} \sum^{m}\left(N^{2} \lambda^{2} h / p\right) \phi(\tau+h / p, z-N \lambda h / p) .
\end{aligned}
$$

Plugging in the Fourier expansion of $\phi$ we get

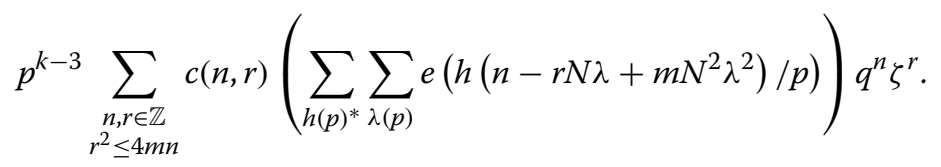

The determination of the inner double sum is a standard computation involving quadratic Gauss sums, so we omit it for brevity. It equals the constant $C_{p}(n, r, m)$ defined above.

The $\sigma$-part of $\phi \mid T_{p}$ is $\chi\left(p^{2}\right)$ times

$$
\begin{aligned}
& \left.p^{k-4} \sum_{\lambda, \mu(p)} \phi\right|_{k, m}\left[\left(\begin{array}{cc}
p & 0 \\
0 & 1 / p
\end{array}\right),[N \lambda, \mu], 1\right] \\
& =p^{k-4} \sum_{\lambda, \mu(p)} p^{k} e^{m}\left(N^{2} \lambda^{2} \tau+2 N \lambda z+N \lambda \mu\right) \phi\left(p^{2} \tau, p(z+N \lambda \tau+\mu)\right) .
\end{aligned}
$$

Since $\phi$ is periodic in the second argument and $\lambda, \mu$ are integers, the sum over $\mu$ gives a factor $p$. Inserting the Fourier expansion we get

$$
=p^{2 k-3} \sum_{\lambda(p)} \sum_{\substack{n, r \in \mathbb{Z} \\ r^{2} \leq 4 m n}} c(n, r) q^{n p^{2}+r p N \lambda+m N^{2} \lambda^{2}} \zeta^{r p+2 m N \lambda} .
$$

We set $n^{\prime}=n p^{2}+r p N \lambda+m N^{2} \lambda^{2}$ and $r^{\prime}=r p+2 m N \lambda$. Then we find $r=\left(r^{\prime}-2 m N \lambda\right) / p$ and, after a short calculation, $n=\left(n^{\prime}-r^{\prime} N \lambda+m N^{2} \lambda^{2}\right) / p^{2}$. Finally, replacing $\lambda$ by $-\lambda$, the last line becomes

$$
=p^{2 k-3} \sum_{\substack{n^{\prime}, r^{\prime} \in \mathbb{Z} \\ r^{\prime 2} \leq 4 m n^{\prime}}} \sum_{\lambda(p)} c\left(\left(n^{\prime}+r^{\prime} N \lambda+m N^{2} \lambda^{2}\right) / p^{2},\left(r^{\prime}+2 m N \lambda\right) / p\right) q^{n^{\prime}} \zeta^{r^{\prime}} .
$$

This completes the proof.

Next, we define a Fricke involution on $J_{k, m}\left(\Gamma_{0}\left(N^{2}\right) \ltimes(N \mathbb{Z} \times \mathbb{Z}), \chi\right)$.

Lemma 2.3. Let $\phi \in J_{k, m}\left(\Gamma_{0}\left(N^{2}\right) \ltimes(N \mathbb{Z} \times \mathbb{Z}), \chi\right)$. Then

$$
\phi\left|W_{N^{2}}:=\phi\right|_{k, m}\left(\begin{array}{cc}
0 & -1 / N \\
N & 0
\end{array}\right)
$$

is in $J_{k, m}\left(\Gamma_{0}\left(N^{2}\right) \ltimes(N \mathbb{Z} \times \mathbb{Z}), \bar{\chi}\right)$ and it holds $\phi\left|W_{N^{2}}\right| W_{N^{2}}=\chi(-1) \phi$. If $\phi$ is a cusp form, so is $\phi \mid W_{N^{2}}$. 


$$
\begin{aligned}
& \text { Proof. For }\left(\begin{array}{ll}
a & b \\
c & d
\end{array}\right) \in \Gamma_{0}\left(N^{2}\right) \text { and }[\lambda, \mu] \in N \mathbb{Z} \times \mathbb{Z} \text { we have } \\
& W_{N^{2}} \cdot\left[\left(\begin{array}{ll}
a & b \\
c & d
\end{array}\right),[\lambda, \mu], 1\right]=\left[\left(\begin{array}{cc}
d & -c / N^{2} \\
-b N^{2} & a
\end{array}\right),[-\mu N, \lambda / N], 1\right] \cdot W_{N^{2}} .
\end{aligned}
$$

The transformation behaviour now follows from $\chi(a)=\bar{\chi}(d)$ as $a d \equiv 1 \bmod N^{2}$. The remaining properties are obvious.

The existence of this nice Fricke involution is the main reason why we work with the group $\Gamma_{0}\left(N^{2}\right) \ltimes(N \mathbb{Z} \times \mathbb{Z})$. It is easy to check that for $(p, 2 m N)=1$ we have the commutation relation

$$
\phi\left|W_{N^{2}}\right| T_{p}=\bar{\chi}\left(p^{2}\right) \phi\left|T_{p}\right| W_{N^{2}} .
$$

We now introduce a twisting and a projection operator.

Lemma 2.4. Let $\phi=\sum_{r^{2} \leq 4 m n} c(n, r) q^{n} \zeta^{r} \in J_{k, m}\left(\Gamma_{0}\left(N^{2}\right) \ltimes(N \mathbb{Z} \times \mathbb{Z}), \chi\right)$. Further, let $p$ be a positive integer.

1 Let $\psi$ be a primitive Dirichlet character mod $p$. Then the twisted function

$$
\phi_{\psi}(\tau, z):=\sum_{\substack{n, r \in \mathbb{Z} \\ r^{2} \leq 4 m n}} \psi\left(r^{2}-4 m n\right) c(n, r) q^{n} \zeta^{r}
$$

is in $J_{k, m}\left(\Gamma_{0}\left(N^{2} p^{2}\right) \ltimes(N p \mathbb{Z} \times \mathbb{Z}), \chi \psi^{2}\right)$.

2 The projected function

$$
\phi \mid B_{p}(\tau, z):=\sum_{\substack{n, r \in \mathbb{Z} \\ r^{2} \leq 4 m n \\ p \mid\left(r^{2}-4 m n\right)}} c(n, r) q^{n} \zeta^{r}
$$

is in $J_{k, m}\left(\Gamma_{0}\left(N^{2} p^{2}\right) \ltimes(N p \mathbb{Z} \times \mathbb{Z}), \chi\right)$.

Both operators map cusp forms to cusp forms.

Proof. First note that the Fourier coefficients of $\phi_{\psi}$ and $\phi \mid B_{p}$ are invariant under $n \mapsto n+r N p \lambda+m N^{2} p^{2} \lambda^{2}, r \mapsto r+2 m N p \lambda$ for $\lambda \in \mathbb{Z}$, which is equivalent to the invariance of $\phi$ under $[\lambda, \mu] \in N p \mathbb{Z} \times \mathbb{Z}$.

We check the transformation behaviour of $\phi_{\psi}$ under $\Gamma_{0}\left(N^{2} p^{2}\right)$. Since $\psi$ is primitive, we can write

$$
\psi(n)=\frac{1}{G(\bar{\psi})} \sum_{\alpha(p)^{*}} \bar{\psi}(\alpha) e(n \alpha / p)
$$


with the Gauss sum $G(\bar{\psi})=\sum_{\alpha(p)^{*}} \bar{\psi}(\alpha) e(\alpha / p)$. We compute

$$
\begin{aligned}
& \phi_{\psi}(\tau, z)= \frac{1}{G(\bar{\psi})} \sum_{\alpha(p)^{*}} \bar{\psi}(\alpha) \sum_{\substack{n, r \in \mathbb{Z} \\
r^{2} \leq 4 m n}} c(n, r) e\left(\left(r^{2}-4 m n\right) \alpha / p\right) q^{n} \zeta^{r} \\
&= \frac{1}{G(\bar{\psi})} \sum_{\alpha(p)^{*}} \bar{\psi}(\alpha) \sum_{\rho(p)} \sum_{\substack{n, r \in \mathbb{Z} \\
r^{2} \leq 4 m n \\
r \equiv \rho(p)}} c(n, r) e\left(\left(r^{2}-4 m n\right) \alpha / p\right) q^{n} \zeta^{r} \\
&= \frac{1}{G(\bar{\psi})} \sum_{\alpha(p)^{*}} \bar{\psi}(\alpha) \sum_{\rho(p)} e\left(\rho^{2} \alpha / p\right) \\
&= \frac{1}{p G(\bar{\psi})} \sum_{\alpha(p)^{*}} \bar{\psi}(\alpha) \sum_{\beta(p)} G(\alpha, \beta, p) \\
& \times\left.\phi(\tau, z)\right|_{m, k}\left[\left(\begin{array}{l}
1-4 m \alpha / p \\
0
\end{array}\right),[0,-\beta / p], 1\right] \\
&\left.\frac{1}{p} \sum_{\beta(p)} e((\rho-r) \beta / p)\right) e(-4 m n \alpha / p) q^{n} \zeta^{r} \\
& 0
\end{aligned}
$$

where $G(\alpha, \beta, p)=\sum_{\rho(p)} e\left(\left(\rho^{2} \alpha+\rho \beta\right) / p\right)$ is a quadratic Gauss sum. For $M=\left(\begin{array}{ll}a & b \\ c & d\end{array}\right) \in$ $\Gamma_{0}\left(N^{2} p^{2}\right)$ we have

$$
\begin{aligned}
& {\left[\left(\begin{array}{cc}
1 & -4 m \alpha / p \\
0 & 1
\end{array}\right),[0,-\beta / p], 1\right] \cdot[M,[0,0], 1]} \\
& =\left[M^{\prime},\left[\lambda^{\prime}, \mu^{\prime}\right], 1\right] \cdot\left[\left(\begin{array}{cc}
1 & -4 m d^{2} \alpha / p \\
0 & 1
\end{array}\right),[0,-d \beta / p], 1\right]
\end{aligned}
$$

where

$$
M^{\prime}=\left(\begin{array}{cc}
a-4 m c \alpha / p & b+4 m d \alpha(a d-1) / p-16 m^{2} c d^{2} \alpha^{2} / p^{2} \\
c & d+4 m c \alpha d^{2} / p
\end{array}\right) \in \Gamma_{0}\left(N^{2} p^{2}\right)
$$

and

$$
\left[\lambda^{\prime}, \mu^{\prime}\right]=[-c \beta / p, 0]\left(\begin{array}{cc}
1 & 4 m d^{2} \alpha / p \\
0 & 1
\end{array}\right) \in N p \mathbb{Z} \times \mathbb{Z} .
$$

Note that $\chi\left(M^{\prime}\right)=\chi(M)$ since $\chi$ is a character $\bmod N^{2}$ and $c / p$ is divisible by $N^{2}$. Let $\bar{d} \in \mathbb{Z}$ with $d \bar{d} \equiv 1 \bmod p$. Replacing $\alpha$ by $\bar{d}^{2} \alpha$ and $\beta$ by $\bar{d} \beta$, and using the elementary property $G\left(\bar{d}^{2} \alpha, \bar{d} \beta, p\right)=G(\alpha, \beta, p)$ of the Gauss sum, we find

$$
\begin{aligned}
& \left.\phi_{\psi}\right|_{k, m} M \\
& =\frac{1}{p G(\bar{\psi})} \chi(M) \sum_{\alpha(p)^{*}} \bar{\psi}(\alpha) \sum_{\beta(p)} G(\alpha, \beta, p) \phi\left(\tau-4 m d^{2} \alpha / p, z-d \beta / p\right) \\
& =\frac{1}{p G(\bar{\psi})} \chi(M) \sum_{\alpha(p)^{*}} \bar{\psi}\left(\bar{d}^{2} \alpha\right) \sum_{\beta(p)} G\left(\bar{d}^{2} \alpha, \bar{d} \beta, p\right) \phi(\tau-4 m \alpha / p, z-\beta / p) \\
& =\chi(M) \psi(d)^{2} \frac{1}{p G(\bar{\psi})} \sum_{\alpha(p)^{*}} \bar{\psi}(\alpha) \sum_{\beta(p)} G(\alpha, \beta, p) \phi(\tau-4 m \alpha / p, z-\beta / p) \\
& =\chi(M) \psi(M)^{2} \phi_{\psi} .
\end{aligned}
$$


This shows that $\phi_{\psi}$ transforms correctly. A standard argument shows that $\phi_{\psi}$ has suitable Fourier expansions at the cusps.

For $\phi \mid B_{p}$ a similiar calculation as above gives

$$
\phi\left|B_{p}(\tau, z)=\frac{1}{p^{2}} \sum_{\alpha(p)} \sum_{\beta(p)} G(\alpha, \beta, p) \phi(\tau, z)\right|_{m, k}\left[\left(\begin{array}{cc}
1 & -4 m \alpha / p \\
0 & 1
\end{array}\right),[0,-\beta / p], 1\right] .
$$

Now we proceed in the same way as before to get the transformation law for $\phi \mid B_{p}$ under $\Gamma_{0}\left(N^{2} p^{2}\right)$.

One could treat twists of Jacobi forms by $\chi(n)$ or $\chi(r)$ in a similar way. The latter, for example, has been considered in [10].

Let $\phi \in J_{k, m}\left(\Gamma_{0}\left(N^{2}\right) \ltimes(N \mathbb{Z} \times \mathbb{Z}), \chi\right)$ and let $\psi$ be a quadratic character mod $p$. For each prime $q$ with $(q, p)=1$ it holds

$$
\begin{aligned}
\left(\phi \mid T_{q}\right)_{\psi} & =\phi_{\psi} \mid T_{q}, \\
\phi\left|T_{q}\right| B_{p} & =\phi\left|B_{p}\right| T_{q} .
\end{aligned}
$$

This can be checked by comparing the actions of the operators on Fourier expansions. The interplay between the Fricke involution and the twisting and projection operators is described by the following lemma.

Lemma 2.5. Let $p$ be a prime with $(p, 2 m N)=1$. For $\phi \in J_{k, m}\left(\Gamma_{0}\left(N^{2}\right) \ltimes(N \mathbb{Z} \times \mathbb{Z}), \chi\right)$ and $\psi=(\dot{\bar{p}})$ we have

$$
\left(\phi \mid W_{N^{2}}^{-1}\right)_{\psi} \mid W_{N^{2} p^{2}}=\bar{\chi}(p) p \sum_{\substack{n, r \in \mathbb{Z} \\ r^{2} \leq 4 m n \\ p \mid n, r}} c(n, r) q^{n} \zeta^{r}-\bar{\chi}(p) \sum_{\substack{n, r \in \mathbb{Z} \\ r^{2} \leq 4 m n \\ p \mid r}} c(n, r) q^{n} \zeta^{r}
$$

and

$$
\phi\left|W_{N^{2}}^{-1}\right| B_{p} \mid W_{N^{2} p^{2}}=p^{k-1} \phi\left(p^{2} \tau, p z\right)+\bar{\chi}(p) \sum_{\substack{n, r \in \mathbb{Z} \\ r^{2} \leq 4 m n \\ p \mid r}}\left(\frac{r^{2}-4 m n}{p}\right) c(n, r) q^{n} \zeta^{r} .
$$

Proof. Using the representation of $\phi_{\psi}$ obtained in the proof of Lemma 2.4 we have

$$
\begin{array}{r}
\left(\phi \mid W_{N^{2}}^{-1}\right)_{\psi} \mid W_{N^{2} p^{2}}=\frac{\psi(-m)}{p G(\bar{\psi})} \sum_{\alpha(p)^{*}} \bar{\psi}(\alpha) \sum_{\beta(p)}\left(\sum_{\rho(p)} e\left(-m\left(\rho^{2} \alpha+2 \rho \beta\right) / p\right)\right) \\
\times\left.\phi\right|_{k, m} W_{N^{2}}^{-1}\left[\left(\begin{array}{cc}
1 & \alpha / p \\
0 & 1
\end{array}\right),[0, \beta / p], 1\right] W_{N^{2} p^{2}},
\end{array}
$$


where we replaced $-4 m \alpha$ by $\alpha,-\beta$ by $\beta$ and $\rho$ by $2 m \rho \bmod p$. Let $\overline{N^{2} \alpha} \in \mathbb{Z}$ denote an inverse of $N^{2} \alpha \bmod p$. We have

$$
\begin{aligned}
& W_{N^{2}}^{-1}\left[\left(\begin{array}{cc}
1 & \alpha / p \\
0 & 1
\end{array}\right),[0, \beta / p], 1\right] W_{N^{2} p^{2}} \\
& =\left[\left(\begin{array}{cc}
p & \overline{N^{2} \alpha} \\
-N^{2} \alpha\left(1-\overline{N^{2} \alpha} N^{2} \alpha\right) / p
\end{array}\right),[N \beta, 0], 1\right] \\
& \quad \cdot\left[\left(\begin{array}{cc}
1 & -\overline{N^{2} \alpha} / p \\
0 & 1
\end{array}\right),\left[0, \overline{N^{2} \alpha} N \beta / p\right], e\left(-\bar{\alpha} \beta^{2} / p\right)\right] .
\end{aligned}
$$

We obtain

$$
\begin{aligned}
\left(\phi \mid W_{N^{2}}^{-1}\right)_{\psi} \mid W_{N^{2} p^{2}}= & \frac{\bar{\chi}(p)}{p G(\bar{\psi})} \psi(-m) \sum_{\alpha(p)^{*}} \bar{\psi}(\alpha) \\
& \times \sum_{\beta(p)}\left(\sum_{\rho(p)} e\left(-m\left(\rho^{2} \alpha+2 \rho \beta+\bar{\alpha} \beta^{2}\right) / p\right)\right) \\
& \times\left.\phi\right|_{k, m}\left[\left(\begin{array}{cc}
1 & -\overline{N^{2} \alpha} / p \\
0 & 1
\end{array}\right),[0, \overline{N \alpha} \beta / p], 1\right] .
\end{aligned}
$$

The sum over $\rho$ is just $\psi(-m \alpha) G(\psi)$. Since $\psi=\bar{\psi}$, we find

$$
\left(\phi \mid W_{N^{2}}^{-1}\right)_{\psi}\left|W_{N^{2} p^{2}}=\frac{\bar{\chi}(p)}{p} \sum_{\alpha(p)^{*} \beta(p)} \sum_{k, m} \phi\right|_{k}\left[\left(\begin{array}{cc}
1 & \alpha / p \\
0 & 1
\end{array}\right),[0, \beta / p], 1\right] .
$$

Plugging in the Fourier expansion of $\phi$ and an easy calculation give the stated formula.

For the projection operator we use the representation for $\phi \mid B_{p}$ given at the end of the proof of Lemma 2.4. The summand for $\alpha=0$ gives $p^{k-1} \phi\left(p^{2} \tau, p z\right)$ and the remaining part can be treated as above.

\section{Proofs of the main results}

We start with the proof of Theorem 1.2 as it will be needed in the proof of Theorem 1.1. Recall that $v_{\ell}(\phi)=\inf _{n, r} v_{\ell}(c(n, r))$.

Proof of Theorem 1.2. First note that $\phi \mid U_{N}(\tau, z)=\phi(\tau, N z)$ is in $J_{k, m N^{2}}\left(\Gamma_{1}\left(N^{2}\right) \ltimes \mathbb{Z}^{2}\right)$ and $\phi\left|U_{N}\right|_{k, m N^{2}} M=\left.\phi\right|_{k, m} M \mid U_{N}$. Since $\left.\phi\right|_{k, m} M$ and $\left.\phi\right|_{k, m} M \mid U_{N}$ have the same set of Fourier coefficients, it suffices to show the claim for $\phi \in J_{k, m N^{2}}\left(\Gamma_{1}\left(N^{2}\right) \ltimes \mathbb{Z}^{2}\right)$. To simplify the notation, we take $\phi \in J_{k, m}\left(\Gamma_{1}(N) \ltimes \mathbb{Z}^{2}\right)$.

Such a form $\phi$ has a theta decomposition

$$
\phi(\tau, z)=\sum_{\mu(2 m)} h_{\mu}(\tau) \theta_{m, \mu}(\tau, z)
$$

with

$$
h_{\mu}(\tau)=\sum_{D<0} c_{\mu}(D) q^{-D / 4 m}, \quad \theta_{m, \mu}(\tau, z)=\sum_{\substack{r \in \mathbb{Z} \\ r \equiv \mu(2 m)}} q^{r^{2} / 4 m} \zeta^{r},
$$


where $c_{\mu}(D)=c\left(\left(\mu^{2}-D\right) / 4 m, \mu\right)$, see [5], §5. The theta function $\theta_{m, \mu}$ satisfies the transformation rules

$$
\begin{aligned}
\theta_{m, \mu}(\tau+1, z) & =e\left(\mu^{2} / 4 m\right) \theta_{m, \mu}, \\
\theta_{m, \mu}\left(-\frac{1}{\tau}, \frac{z}{\tau}\right) & =\sqrt{\tau / 2 m i} e\left(m z^{2} / \tau\right) \sum_{\nu(2 m)} e(-\mu \nu / 2 m) \theta_{m, \nu} .
\end{aligned}
$$

This together with the transformation behaviour of $\phi$ under $\Gamma_{1}(N)$ implies that the tuple $\left(h_{\mu}\right)_{\mu(2 m)}$ transforms under $\Gamma_{1}(N)$ as a vector valued modular form of weight $k-1 / 2$ for the Weil representation $\rho_{L}$ of the lattice $L=\mathbb{Z}$ with quadratic form $q(x)=$ $-m x^{2}$, compare [2], Section 1.1. For $M \in S L_{2}(\mathbb{Z})$ we have

$$
\left(\left.\phi\right|_{k, m} M\right)(\tau, z)=\sum_{\mu(2 m)}\left(\left.h_{\mu}\right|_{k-1 / 2} M\right)(\tau) \cdot\left(\left.\theta_{m, \mu}\right|_{1 / 2, m} M\right)(\tau, z) .
$$

From the transformation rules of $\theta_{m, \mu}$ stated above it follows that $\left.\theta_{m, \mu}\right|_{1 / 2, m} M=$ $\sum_{v(2 m)} \rho_{v, \mu} \theta_{m, v}$ with some algebraic numbers $\rho_{v, \mu}$ with non-negative $\ell$-adic valuation. Here the assumption $(\ell, 2 m N)=1$ was used. Thus to prove the claim of the theorem it remains to show that the functions $\left.h_{\mu}\right|_{k-1 / 2} M$ have algebraic coefficients with $v_{\ell}\left(\left.h_{\mu}\right|_{k-1 / 2} M\right) \geq v_{\ell}\left(h_{\mu}\right)$.

By the explicit formula for the action of $\Gamma_{1}(4 m)$ in the Weil representation $\rho_{L}$ given in [12], Proposition 4.5., the functions $h_{\mu}$ are modular forms of weight $k-1 / 2$ for $\Gamma_{1}(4 m N)$ and character $\chi_{\mu}\left(\left(\begin{array}{ll}a & b \\ c & d\end{array}\right)\right)=e\left(-b \mu^{2} / 4 m\right)$. We write

$$
\left.h_{\mu}\right|_{k-1 / 2} M=\left.\left(\left.h_{\mu}\right|_{k-1 / 2}\left(\begin{array}{cc}
2 m & 0 \\
0 & \frac{1}{2 m}
\end{array}\right)\right)\right|_{k-1 / 2}\left(\begin{array}{cc}
\frac{1}{2 m} & 0 \\
0 & 2 m
\end{array}\right) M .
$$

The inner function is now a modular form of weight $k-1 / 2$ for $\Gamma_{1}\left(16 m^{3} N\right)$ and trivial character with algebraic Fourier coefficients and the same $\ell$-adic valuation as $h_{\mu}$. The product of the remaining two matrices can be written as $M^{\prime}\left(\begin{array}{ll}\alpha & \beta \\ 0 & \delta\end{array}\right)$ with $M^{\prime} \in S L_{2}(\mathbb{Z})$ and $\alpha, \beta, \delta \in \mathbb{Q}$ with $v_{\ell}(\delta)=0$. It now follows from the $q$-expansion principle (see [7], Corollary 1.6.2., or [4], Remark 12.3.5., for the $q$-expansion principle for integral weight modular forms, and the proof of Lemma 1 in [1] for the transition to half-integral weight forms) that $\left.h_{\mu}\right|_{k-1 / 2} M$ has algebraic coefficients with $v_{\ell}\left(\left.h_{\mu}\right|_{k-1 / 2} M\right) \geq v_{\ell}\left(h_{\mu}\right)$. The assumption $(\ell, 2 m N)=1$ is again needed here.

Proposition 3.1. Let $p$ be a prime with $(p, 2 m N)=1$ and let $\psi=(\dot{\bar{p}})$. Suppose that $\phi \in J_{k, m}\left(\Gamma_{0}\left(N^{2}\right) \ltimes(N \mathbb{Z} \times \mathbb{Z}), \chi\right)$ has integral algebraic Fourier coefficients. Let $\ell$ be a prime with $(\ell, 2 m N p(p-1))=1$, let $\varepsilon \in\{ \pm 1\}$ and let

$$
h:=\phi \mid\left(1-B_{p}\right)-\varepsilon \phi_{\psi}=2 \sum_{\substack{n, r \in \mathbb{Z} \\ \psi\left(r^{2}-4 m n\right)=-\varepsilon}} c(n, r) q^{n} \zeta^{r} .
$$

Then $v_{\ell}\left(\phi \mid T_{p}-\chi(p) \varepsilon\left(p^{k-1}+p^{k-2}\right) \phi\right) \geq v_{\ell}(h)$. In particular, if $\phi$ is an eigenform of $T_{p}$ with eigenvalue $\lambda_{p}$ and $v_{\ell}(\phi)=0$, there is a discriminant $D=r^{2}-4 m n<0$ such that $\left(\frac{D}{p}\right)=-\varepsilon$ and $\nu_{\ell}(c(n, r)) \leq \nu_{\ell}\left(\lambda_{p}-\chi(p) \varepsilon\left(p^{k-1}+p^{k-2}\right)\right)$. 
Proof. Let $h^{\prime}=h \mid W_{N^{2} p^{2}}$. If we write $\left(\begin{array}{cc}0 & -1 / N p \\ N p & 0\end{array}\right)=\left(\begin{array}{cc}0 & -1 \\ 1 & 0\end{array}\right)\left(\begin{array}{cc}N p & 0 \\ 0 & 1 / N p\end{array}\right)$,

Theorem 1.2 implies that $h^{\prime}$ has algebraic coefficients with $v:=v_{\ell}(h)=v_{\ell}\left(h^{\prime}\right)$. We get equality instead of an inequality here since $W_{N^{2} p^{2}}$ is an involution (up to a sign). Further, let $c^{\prime}(n, r)$ denote the Fourier coefficients of $\phi^{\prime}=\phi \mid W_{N^{2}}$, which are again algebraic. We write

$$
h^{\prime}=\phi^{\prime}\left|W_{N^{2}}^{-1}\right| W_{N^{2} p^{2}}-\phi^{\prime}\left|W_{N^{2}}^{-1}\right| B_{p}\left|W_{N^{2} p^{2}}-\varepsilon\left(\phi^{\prime} \mid W_{N^{2}}^{-1}\right)_{\psi}\right| W_{N^{2} p^{2}} .
$$

It is easily checked that $\phi^{\prime}\left|W_{N^{2}}^{-1}\right| W_{N^{2} p^{2}}(\tau, z)=p^{k} \phi^{\prime}\left(p^{2} \tau, p z\right)$. Since $\phi^{\prime}$ transforms with character $\bar{\chi}$, we get from Lemma 2.5 the formula

$$
\begin{gathered}
h^{\prime}=\left(p^{k}-p^{k-1}\right) \sum_{\substack{n, r \in \mathbb{Z} \\
r^{2} \leq 4 m n}} c^{\prime}\left(n / p^{2}, r / p\right) q^{n} \zeta^{r}-\chi(p) \sum_{\substack{n, r \in \mathbb{Z} \\
r^{2} \leq 4 m n \\
p \mid r}}\left(\frac{D}{p}\right) c^{\prime}(n, r) q^{n} \zeta^{r} \\
-\chi(p) \varepsilon p \sum_{\substack{n, r \in \mathbb{Z} \\
r^{2} \leq 4 m n \\
p \mid n, r}} c^{\prime}(n, r) q^{n} \zeta^{r}+\chi(p) \varepsilon \sum_{\substack{n, r \in \mathbb{Z} \\
r^{2} \leq 4 m n \\
p \mid r}} c^{\prime}(n, r) q^{n} \zeta^{r},
\end{gathered}
$$

where $D=r^{2}-4 m n$. This gives the relations

$$
\begin{array}{rlrl}
v_{\ell}\left(\left(\frac{D}{p}\right) c^{\prime}(n, r)-\varepsilon c^{\prime}(n, r)\right) & \geq v, & & \text { if } p \mid r, p \nmid n, \\
v_{\ell}\left(c^{\prime}(n, r)\right) \geq v, & & \text { if } p|r, p| n, p^{2} \nmid n, \\
\nu_{\ell}\left(c^{\prime}(n, r)-\bar{\chi}(p) \varepsilon p^{k-1} c^{\prime}\left(n / p^{2}, r / p\right)\right) \geq v, & & \text { if } p\left|r, p^{2}\right| n,
\end{array}
$$

where we divided by $\chi(p), \varepsilon, p^{k-1}$ or $p-1$ at some places. Using $c^{\prime}(n, r)=c^{\prime}(n+r N \lambda+$ $\left.m N^{2} \lambda^{2}, r+2 m N \lambda\right)$ for $\lambda \in \mathbb{Z}$ and the assumption $(p, 2 m N)=1$ we may assume that $p \mid r$. For $p \mid r$ and $(p, 2 m N)=1$, the coefficient $c^{* *}(n, r)$ of $\phi^{\prime} \mid T_{p}$ is given by

$$
c^{* *}(n, r)=c^{\prime}\left(p^{2} n, p r\right)+\bar{\chi}(p)\left(\frac{D}{p}\right) p^{k-2} c^{\prime}(n, r)+\bar{\chi}\left(p^{2}\right) p^{2 k-3} c^{\prime}\left(n / p^{2}, r / p\right) .
$$

Using the relations (2), (3), (4) it is straightfoward to verify that

$$
v_{\ell}\left(c^{\prime *}(n, r)-\bar{\chi}(p) \varepsilon\left(p^{k-1}+p^{k-2}\right) c^{\prime}(n, r)\right) \geq v .
$$

The result now follows from $\phi^{\prime}\left|T_{p}=\phi\right| W_{N^{2}}^{-1}\left|T_{p}=\bar{\chi}\left(p^{2}\right) \phi\right| T_{p} \mid W_{N^{2}}^{-1}$ and Theorem 1.2.

Proposition 3.2. Let $p$ be a prime with $(p, 2 m N)=1$ and let $\phi \in J_{k, m}\left(\Gamma_{0}\left(N^{2}\right) \ltimes\right.$ $(N \mathbb{Z} \times \mathbb{Z}), \chi)$ with integral algebraic Fourier coefficients which is an eigenform of $T_{p}$ with eigenvalue $\lambda_{p}$. Let $\ell$ be a prime with $(\ell, 2 m N p(p-1))=1$ and let $\varepsilon \in\{ \pm 1\}$. Suppose that there is some discriminant $D^{*}=r^{* 2}-4 m n^{*}<0$ with $\left(\frac{D^{*}}{p}\right)=\varepsilon$ and $v_{\ell}\left(c\left(n^{*}, r^{*}\right)\right)=0$. Then there exist infinitely many discriminants $D=r^{2}-4 m n<0$ with pairwise distinct square free parts and $\left(\frac{D}{p}\right)=-\varepsilon$ such that $v_{\ell}(c(n, r)) \leq v_{\ell}\left(\lambda_{p}-\chi(p) \varepsilon\left(p^{k-1}+p^{k-2}\right)\right)$.

Proof. We prove by induction on $t$ that there are negative discriminants $D_{1}=r_{1}^{2}-$ $4 m n_{1}, \ldots, D_{t}=r_{t}^{2}-4 m n_{t}$ with pairwise distinct square free parts such that $\left(\frac{D_{j}}{p}\right)=-\varepsilon$ and $v_{\ell}\left(c\left(n_{j}, r_{j}\right)\right) \leq v_{\ell}\left(\lambda_{p}-\chi(p) \varepsilon\left(p^{k-1}+p^{k-2}\right)\right)$ for $j=1, \ldots, t$.

For $t=1$ the statement follows from the last proposition. Now let us assume that for $\phi$ as above and fixed $t>1$ we can always find $t-1$ discriminants $D_{2}, \ldots, D_{t}$ with the 
desired properties. By the last proposition we find a discriminant $D_{1}=r_{1}^{2}-4 m n_{1}<0$ with $\left(\frac{D_{1}}{p}\right)=-\varepsilon$ and $\nu_{\ell}\left(c\left(n_{1}, r_{1}\right)\right) \leq \nu_{\ell}\left(\lambda_{p}-\chi(p) \varepsilon\left(p^{k-1}+p^{k-2}\right)\right)$. Since $\left(\frac{D_{1}}{p}\right)=-\varepsilon$ and $\left(\frac{D^{*}}{p}\right)=\varepsilon$ the square free parts of $D_{1}$ and $D^{*}$ are different. Thus we can choose a prime $q$ with

$$
(q, \ell)=1, \quad(q, p)=1, \quad\left(\frac{D^{*}}{q}\right)=+\varepsilon, \quad\left(\frac{D_{1}}{q}\right)=-\varepsilon
$$

Put $\psi=(\dot{\bar{q}})$ and consider the function

$$
\phi_{1}=\frac{1}{2}\left(\phi \mid\left(1-B_{q}\right)+\varepsilon \phi_{\psi}\right) \in J_{k, m}\left(\Gamma_{0}\left(N^{2} q^{2}\right) \ltimes(N q \mathbb{Z} \times \mathbb{Z}), \chi \psi^{2}\right) .
$$

Then $\phi_{1}$ has the Fourier expansion

$$
\phi_{1}=\sum_{\substack{n, r \in \mathbb{Z} \\ r^{2} \leq 4 m n}} c_{1}(n, r) q^{n} \zeta^{r}, \quad \text { with } c_{1}(n, r)= \begin{cases}c(n, r), & \text { if }\left(\frac{r^{2}-4 m n}{q}\right)=\varepsilon, \\ 0, & \text { otherwise. }\end{cases}
$$

Since $T_{p}$ commutes with the twisting and projection operators in the definition of $\phi_{1}$, the function $\phi_{1}$ is also an eigenform of $T_{p}$ with eigenvalue $\lambda_{p}$. Further, $c_{1}\left(n^{*}, r^{*}\right)=$ $c\left(n^{*}, r^{*}\right)$, so $v_{\ell}\left(\phi_{1}\right)=v_{\ell}(\phi)=0$. We can apply the induction hypothesis on $\phi_{1}$ to find discriminants $D_{2}=r_{2}^{2}-4 m n_{2}, \ldots, D_{t}=r_{t}^{2}-4 m n_{t}$ with pairwise distinct square free parts such that $\left(\frac{D_{j}}{p}\right)=-\varepsilon$ and $v_{\ell}\left(c\left(n_{j}, r_{j}\right)\right)=v_{\ell}\left(c_{1}\left(n_{j}, r_{j}\right)\right) \leq v_{\ell}\left(\lambda_{p}-\chi(p) \varepsilon\left(p^{k-1}+\right.\right.$ $\left.p^{k-2}\right)$ ). Since $\left(\frac{D_{1}}{q}\right)=-\varepsilon$, we have $c_{1}(n, r)=0$ for all $n, r$ such that $r^{2}-4 m n$ has the same square free part as $D_{1}$, so the square free parts of $D_{2}, \ldots, D_{t}$ are different from the square free part of $D_{1}$. This completes the proof.

So far, the discriminants given in Proposition 3.2 need not be fundamental. To find fundamental discriminants with the properties as in the proposition, we will make use of the relations given by the Hecke eigenform equations.

Lemma 3.3. Let $\phi \in J_{k, m}\left(\Gamma_{0}\left(N^{2}\right) \ltimes(N \mathbb{Z} \times \mathbb{Z})\right.$, $\left.\chi\right)$ be an eigenform of all Hecke operators $T_{p},(p, m N)=1$, with algebraic Fourier coefficients $c(n, r)$. Let $\ell$ be a prime and $D=$ $r^{2}-4 m n \leq 0$ a fundamental discriminant. Then $v_{\ell}\left(c\left(f^{2} n, f r\right)\right) \geq v_{\ell}(c(n, r))$ for all $f \in \mathbb{Z}$ with $(f, m N)=1$.

Proof. By induction on $|f|$ : For $f=1$ there is nothing to show and for $f=-1$ we have $c(n, r)=(-1)^{k} c(n,-r)$. For $|f|>1$ we choose a prime divisor $p$ of $f$ and write $f=p f_{1}$. The action of $T_{p}$ on $\phi$ gives the relation

$$
\begin{aligned}
& \lambda_{p} c\left(f_{1}^{2} n, f_{1} r\right) \\
& =c\left(f^{2} n, f r\right)+\chi(p)\left(\frac{f_{1}^{2} D}{p}\right) p^{k-2} c\left(f_{1}^{2} n, f_{1} r\right) \\
& \quad+\chi\left(p^{2}\right) p^{2 k-3} \sum_{\lambda(p)} c\left(\left(f_{1}^{2} n+f_{1} r N \lambda+m N^{2} \lambda^{2}\right) / p^{2},\left(f_{1} r+2 m N \lambda\right) / p\right) .
\end{aligned}
$$


We find

$$
\begin{aligned}
& v_{\ell}\left(c\left(f^{2} n, f r\right)\right) \\
& \geq \min \left(v_{\ell}\left(\lambda_{q} c\left(f_{1}^{2} n, f_{1} r\right)\right), v_{\ell}\left(p^{k-2} c\left(f_{1}^{2} n, f_{1} r\right)\right),\right. \\
& \left.\quad v_{\ell}\left(p^{2 k-3} \sum_{\lambda(p)} c\left(\left(f_{1}^{2} n+f_{1} r N \lambda+m N^{2} \lambda^{2}\right) / p^{2},\left(f_{1} r+2 m N \lambda\right) / p\right)\right)\right) .
\end{aligned}
$$

In the sum over $\lambda$ the summands can be nonzero only if $p \mid f_{1}$ since $D$ is fundamental, in which case the sum is just $c\left(\left(f_{1} / p\right)^{2} n,\left(f_{1} / p\right) r\right)$ as $(p, m N)=1$. Note that $\lambda_{p}$ is an algebraic integer, so all the terms in the minimum are at least $\nu_{\ell}(c(n, r))$ by the induction hypothesis, which proves the claim.

We would now like to write each $D=r^{2}-4 m n$ in Proposition 3.2 as $D=f^{2} D_{0}$ with a fundamental discriminant $D_{0}=r_{0}^{2}-4 m n_{0}$ and $f \in \mathbb{Z}$, and then use the relations given by the eigenform equations to show that $v_{\ell}\left(c\left(n_{0}, r_{0}\right)\right) \leq v_{\ell}(c(n, r))$. But in general, $D_{0}$ will not necessarily be a square $\bmod 4 m$, as the example $m=3$ and $D=0^{2}-4 \cdot 3 \cdot 3=$ $9 \cdot(-4)$ shows, and even if $D_{0}$ is a square mod $4 m$, the eigenform equations will in general not give information about the relation of $c(n, r)$ and $c\left(f^{2} n_{0}, f r_{0}\right)$ if $r \not \equiv f r_{0}$ $\bmod 2 m$. But these problems can only arise if $(D, m N)>1$, as the following lemma shows.

Lemma 3.4. Let $\phi \in J_{k, m}\left(\Gamma_{0}\left(N^{2}\right) \ltimes(N \mathbb{Z} \times \mathbb{Z})\right.$, $\left.\chi\right)$ with Fourier coefficients $c(n, r)$. Let $D=r^{2}-4 m n<0$ be a discriminant with $(D, m N)=1$ and write $D=f^{2} D_{0}$ with a fundamental discriminant $D_{0}<0$ and some $f \in \mathbb{Z}$. Then $D_{0}$ is a square $\bmod 4 m$, i.e. $D_{0}=r_{0}^{2}-4 m n_{0}$ for some $r_{0}, n_{0} \in \mathbb{Z}$, and $c(n, r)=c\left(f^{2} n_{0}, f r_{0}\right)$.

Proof. It suffices to show the claim in the case that $f=p$ is a prime with $(p, m N)=1$ and $D_{0}<0$ an arbitrary (i.e. not necessarily fundamental) discriminant. We will use that $c(n, r)=c\left(n+r N \lambda+m N^{2} \lambda^{2}, r+2 m N \lambda\right)$ holds for every $\lambda \in \mathbb{Z}$.

Assume that $p$ is odd. Since $2 m N$ is invertible $\bmod p$ we can achieve $r+2 m N \lambda \equiv$ $0(p)$, so we can assume that $p \mid r$. Write $r=p r_{0}$. Then $p^{2} r_{0}^{2}-4 m n=p^{2} D_{0}$ implies $p^{2} \mid 4 m n$, and $(p, 2 m)=1$ shows $p^{2} \mid n$, i.e. $n=p^{2} n_{0}$.

We leave the case $p=2$ to the reader.

If $\phi \in J_{k, m}\left(\Gamma_{0}\left(N^{2}\right) \ltimes(N \mathbb{Z} \times \mathbb{Z}), \chi\right)$ is a Jacobi form with integral algebraic coefficients which is an eigenfunction of all Hecke operators $T_{p}$ for $(p, m N)=1$ with corresponding eigenvalues $\lambda_{p}$, we define the set

$$
\begin{gathered}
A\left(p, \lambda_{p}\right)=\left\{\ell \text { prime }: v_{\ell}\left(\lambda_{p}-\chi(p) \varepsilon\left(p^{k-1}+p^{k-2}\right)\right)>0 \text { for some } \varepsilon \in\{ \pm 1\}\right\} \\
\cup\{\ell \text { prime }: \ell \mid p(p-1)\} .
\end{gathered}
$$

The following result is a more precise variant of Theorem 1.1 from the introduction.

Theorem 3.5. Let $\phi \in J_{k, m}\left(\Gamma_{0}\left(N^{2}\right) \ltimes(N \mathbb{Z} \times \mathbb{Z}), \chi\right)$ be a Jacobi form with integral algebraic coefficients which is an eigenfunction of all Hecke operators $T_{p}$ for $(p, m N)=1$. Let $S=\left\{p_{1}, \ldots, p_{s}\right\}$ be a non-empty finite set of odd primes which are coprime to $m N$ and let $\varepsilon_{1}, \ldots, \varepsilon_{s} \in\{ \pm 1\}$. Let $\ell$ be a prime with $(\ell, 2 m N)=1, \ell \notin A\left(p_{j}, \lambda_{p_{j}}\right)$ for all $p_{j} \in S$ 
and $v_{\ell}\left(c\left(n^{*}, r^{*}\right)\right)=0$ for some $D^{*}=r^{* 2}-4 m n^{*}<0$ with $\left(D^{*}, m N\right)=1$. Then there exist infinitely many fundamental discriminants $D=r^{2}-4 m n<0$ prime to $m N$ such that $\left(\frac{D}{p_{j}}\right)=\varepsilon_{j}$ for all $p_{j} \in S$ and $v_{\ell}(c(n, r))=0$.

Proof. By Lemma 2.4 the projected function

$$
\phi^{\prime}=\phi \mid \prod_{p \mid m N}\left(1-B_{p}\right)=\sum_{\substack{n, r \in \mathbb{Z} \\ r^{2}<m m \\(r, m N)=1}} c(n, r) q^{n} \zeta^{r}
$$

is a Jacobi form for $\Gamma_{0}\left(m^{2} N^{2}\right) \ltimes(m N \mathbb{Z} \times \mathbb{Z})$ with integral algebraic Fourier coefficients, and we have $v_{\ell}\left(\phi^{\prime}\right)=0$ by the assumption on $D^{*}$. Further, $\phi^{\prime}$ is still an eigenfunction of all Hecke operators $T_{p}$ with $(p, m N)=1$.

A repeated application of Proposition 3.1 shows that the function

$$
\phi^{\prime \prime}=\phi^{\prime} \mid \prod_{j=1}^{s-1}\left(1-B_{p_{j}}+\varepsilon_{j}(\cdot)_{\psi_{j}}\right)=\sum_{\substack{n, r \in \mathbb{Z} \\(r, m N)=1 \\\left(\frac{D}{p_{j}}\right)=\varepsilon j, 1 \leq j \leq s-1}} c(n, r) q^{n} \zeta^{r}
$$

(with $\psi_{j}=\left(\frac{\dot{p_{j}}}{)}\right)$ ) again satisfies $v_{\ell}\left(\phi^{\prime \prime}\right)=0$ and is an eigenform of $T_{p}$ for $p$ prime to $m N$ and $p_{1}, \ldots, p_{s-1}$.

If we now apply Proposition 3.2 to $\phi^{\prime \prime}$ with $p=p_{s}$ and $\varepsilon=-\varepsilon_{s}$, we find infinitely many discriminants $D=r^{2}-4 m n<0$ prime to $m N$ with distinct square free parts such that $\left(\frac{D}{p_{j}}\right)=\varepsilon_{j}$ for all $j=1, \ldots, s$ and $v_{\ell}(c(n, r))=0$. Writing each of these discriminants as $D=f^{2} D_{0}$ with necessarily pairwise distinct fundamental discriminants $D_{0}$, Lemma 3.4 shows that $D_{0}=r_{0}^{2}-4 m n_{0}$ is a square mod $4 m$ with $c(n, r)=c\left(f^{2} n_{0}, f r_{0}\right)$, and the eigenform relations from Lemma 3.3 give $v_{\ell}\left(c\left(n_{0}, r_{0}\right)\right) \leq v_{\ell}(c(n, r))=0$, i.e. $v_{\ell}\left(c\left(n_{0}, r_{0}\right)\right)=0$. This completes the proof.

Now we restrict to $N=1$. In order to infer Theorem 1.1 from the last theorem, it only remains to show that for a Hecke eigenform $\phi \in J_{k, m}$ (with $k>2$ if $\phi$ is not a cusp form) and some $p$ with $(p, 2 m)=1$ the set $A\left(p, \lambda_{p}\right)$ is finite, or equivalently, $\lambda_{p} \neq \pm\left(p^{k-1}+p^{k-2}\right)$.

First suppose that $\phi \in J_{k, m}^{\text {cusp }}$ is a cusp form. By [15], Theorem $5, J_{k, m}^{\text {cusp }}$ is Heckeequivariantly isomorphic to a certain subspace of $S_{2 k-2}(m)$, so $\lambda_{p}$ is also an eigenvalue of the Hecke operator $T_{p}$ on $S_{2 k-2}(m)$. Now [8] gives the estimate $\left|\lambda_{p}\right|<p^{k-1}+p^{k-2}$.

If $0 \neq \phi \in J_{k, m}$ is a non-cuspidal Jacobi form of weight $k>2$ and an eigenform of all Hecke operators $T_{p}$ for $(p, m)=1$, then by Theorem 4.4 in [5] the form $\phi$ lies in the space spanned by the $\left(U_{d} \circ V_{d^{\prime}}\right)$-images of Eisenstein series $E_{k, m^{\prime}}^{(\chi)}$ of index $m^{\prime}=f^{2}$ defined after Theorem 2.4 in [5], where $\chi$ runs through the primitive Dirichlet characters $\bmod f$ with $\chi(-1)=(-1)^{k}$. It was shown at the end of the proof of Theorem 2 in [15] that $E_{k, m^{\prime}}^{(\chi)}$ is an eigenform of $T_{p}$ for $\left(p, m^{\prime}\right)=1$ with eigenvalue $\sigma_{2 k-3, \chi}(p)=\sum_{d \mid p} d^{2 k-3} \bar{\chi}(d) \chi(p / d)$. Hence the eigenvalues of $\phi$ are also of this form, and for $k>2$ we have $\sigma_{2 k-3, \chi}(p) \neq \pm\left(p^{k-1}+p^{k-2}\right)$. 
Thus the finite set of primes $\ell$ with $(\ell, 2 m)=1$ for which Theorem 1.1 does not hold is given by the primes in

$$
\bigcap_{\substack{p \text { prime } \\(p, 2 m)=1}} A\left(p, \lambda_{p}\right) \cup\left\{\ell \text { prime }: v_{\ell}(c(n, r))>0 \text { for all } r, n \text { s.t. }(r, m)=1\right\} \text {. }
$$

Now let $\phi \in J_{k, m}^{\text {new }}$ be a newform in the sense of [5] which is an eigenform of all Hecke operators $T_{p}$ with $(p, m)=1$. Then $\phi$ is also an eigenform of the Atkin-Lehner involutions $w_{m^{\prime}}$ for exact divisors $m^{\prime}$ of $m$, see [5], Theorem 5.2. The following proposition shows that if $\phi$ is a newform, we can replace in Theorem 3.5 the requirement that there be a discriminant $D^{*}=r^{* 2}-4 m n^{*}<0$ prime to $m$ with $v_{\ell}\left(c\left(n^{*}, r^{*}\right)\right)=0$ by a condition on the Atkin-Lehner eigenvalues of $\phi$.

Proposition 3.6. Let $\phi \in J_{k, m}^{\text {new }}$ be a Jacobi newform with integral algebraic coefficients which is an eigenfunction of all Hecke operators $T_{p}$ for $(p, m)=1$. Let $\ell$ be a prime with $(\ell, 2 m)=1$ and $v_{\ell}(\phi)=0$ such that $\ell$ does not divide $p-\epsilon_{p}$ for any prime $p \| m$, where $\epsilon_{p} \in\{ \pm 1\}$ denotes the eigenvalue of $\phi$ under the Atkin-Lehner involution $w_{p}$. Then there exists a discriminant $D^{*}=r^{* 2}-4 m n^{*}<0$ prime to $m$ such that $v_{\ell}\left(c\left(n^{*}, r^{*}\right)\right)=0$.

Proof. The proof goes along the same lines as the proof of Lemma 3.1 in [15]: Assume that $v_{\ell}(c(n, r))>0$ for all $r^{2}<4 m n$ with $(r, m)=1$. Using the formula $\sum_{t \mid m} \mu(m) \prod_{p \mid t} e(r / p)=0$ for $(r, m)>1$ the assumption gives

$$
v_{\ell}\left(\left.\sum_{t \mid m} \mu(t) \prod_{p \mid t} \phi\right|_{m}\left[0, \frac{g}{p}\right]\right)>0
$$

for every integer $g$. Let $f(\tau, z)$ denote the function in the braces. It is easily checked that $f$ is a Jacobi form for the group $\Gamma_{1}\left(m^{2}\right) \ltimes(m \mathbb{Z} \times \mathbb{Z})$ with integral algebraic Fourier coefficients. Theorem 1.2 shows that for $M \in S L_{2}(\mathbb{Z})$ the function $\left.f\right|_{k, m} M$ has algebraic Fourier coefficients and $v_{\ell}\left(\left.f\right|_{k, m} M\right) \geq v_{\ell}(f)>0$. Now the calculation in the proof of Lemma 3.1 in [15] gives

$$
v_{\ell}\left(\phi \mid\left(\prod_{d^{2} \mid m} \frac{\mu(d)}{d} u_{d} U_{d}\right) \circ\left(\prod_{p \| m}\left(1-\frac{1}{p} w_{p}\right)\right)\right)>0
$$

where $u_{d}$ is the auxiliary operator from [15], line (6), and $\phi \mid U_{d}(\tau, z)=\phi(\tau, d z)$. Since $\phi$ is a newform and an eigenform of all Hecke operators, we have $\phi \mid u_{d}=0$ for all $d^{2} \mid m$ with $d>1$. In particular, we find

$$
v_{\ell}\left(\phi \mid \prod_{p \| m}\left(1-\frac{1}{p} w_{p}\right)\right)>0 .
$$

The inner function is just $\prod_{p \| m}\left(1-\epsilon_{p} / p\right) \phi$, so we get $v_{\ell}(\phi)>0$, contradicting the assumption $v_{\ell}(\phi)=0$. Hence there is some $D^{*}=r^{* 2}-4 m n^{*}<0$ prime to $m$ with $v_{\ell}\left(c\left(n^{*}, r^{*}\right)\right)=0$. 
Received: 9 July 2015 Accepted: 29 December 2015

Published online: 14 February 2016

\section{References}

1. Bruinier, JH: Nonvanishing modulo $\ell$ of Fourier coefficients of half-integral weight modular forms. Duke Math. J. 98 595-611 (1999)

2. Bruinier, JH: Borcherds Products on $O(2, l)$ and Chern Classes of Heegner Divisors. Lecture Notes in Mathematics, vol. 1780. Springer, Berlin Heidelberg (2002)

3. Carlitz, L: Arithmetic properties of generalized Bernoulli numbers. J. Reine Angew. Math. 202, 174-182 (1959)

4. Diamond, F, Im, J: Modular forms and modular curves. Can. Math. Soc. Conf. Proc. 17, 39-132 (1995)

5. Eichler, M, Zagier, D: The Theory of Jacobi Forms. Birkhäuser, Boston-Basel-Stuttgart (1985)

6. Gross, B, Kohnen, W, Zagier, D: Heegner points and derivatives of L-series. II. Mathematische Ann. 278, 497-562 (1987)

7. Katz, N: $p$-adic properties of modular schemes and modular forms. Modular Funct. One Variable, III (Proc. Internat. Summer School, Univ. Antwerp, Antwerp, 1972), Lecture Notes in Math. 350, 69-190 (1973)

8. Kohnen, W: A simple remark on eigenvalues of Hecke operators on Siegel modular forms. Abh. Math. Sem. Univ. Hamburg. 57, 33-36 (1986)

9. Kohnen, W, Ono, K: Indivisibility of class numbers of imaginary quadratic fields and orders of Tate-Shafarevich groups of elliptic curves with complex multiplication. Invent. Math. 135(2), 387-398 (1999)

10. Martin, Y, Osses, D: On the analogue of Weil's converse theorem for Jacobi forms and their lift to half-integral weight modular forms. Ramanujan J. 26, 155-183 (2011)

11. Ono, K, Skinner, C: Fourier coefficients of half-integral weight modular forms modulo $\ell$. Ann. Math. 147, 453-470 (1998)

12. Scheithauer, NR: The Weil representation of $S L_{2}(\mathbb{Z})$ and some applications. Int Math Res Notices. 2009(8), 1488-1545 (2009)

13. Shimura, G: On modular forms of half-integral weight. Ann. Math. 97, 440-481 (1973)

14. Skoruppa, N-P: Developments in the theory of Jacobi forms. In: Kuznetsov, N, Bykovsky, V (eds.) International Conference on Automorphic Functions and their Applications, Khabarovsk, 27 June - 4 July 1988, pp. 167-185, The USSR Academy of Science, Khabarovsk', (1990)

15. Skoruppa, N-P, Zagier, D: Jacobi forms and a certain space of modular forms. Inventiones Mathematicae. 94 113-146 (1988)

16. Waldspurger, J-L: Sur les coefficients de Fourier des formes modulaires de poids demi-entier. J. Math. Pures Appl. 60 375-484 (1981)

\section{Submit your manuscript to a SpringerOpen ${ }^{\circ}$ journal and benefit from:}

- Convenient online submission

Rigorous peer review

- Immediate publication on acceptance

- Open access: articles freely available online

- High visibility within the field

- Retaining the copyright to your article

Submit your next manuscript at $\boldsymbol{\triangleright}$ springeropen.com 